\title{
Mitochondrial abnormalities drive cell death in Wolfram syndrome 2
}

\author{
Tomotake Kanki ${ }^{1}$, Daniel J Klionsky² \\ ${ }^{1}$ Department of Clinical Chemistry and Laboratory Medicine, Kyushu University Graduate School of Medical Sciences, 3-1-1 Mai- \\ dashi Higashi-ku, Fukuoka 812-8582 Japan; ${ }^{2}$ Life Sciences Institute and Departments of Molecular, Cellular and Developmental \\ Biology and Biological Chemistry, University of Michigan, 210 Washtenaw Ave. Ann Arbor, Michigan 48109-2216 USA \\ Cell Research (2009) 19:922-923. doi: 10.1038/cr.2009.94; published online 3 August 2009
}

Wolfram syndrome (WFS; MIM 222300) is an autosomal recessive disorder with highly variable clinical manifestations. It is characterized by diabetes insipidus, diabetes mellitus, optic atrophy, and deafness (thus, known as DIDMOAD syndrome) [1]. Other neurological and endocrine manifestations include dementia, psychiatric illnesses, renal-tract abnormalities, and bladder atony [2]. Gene linkage and positional cloning analysis reveal that a subset of Wolfram syndrome patients belonging to the WFS1 group (MIM 606201) carry a loss-of-function mutation in the WFS1 gene, which encodes a transmembrane protein, Wolframin, localizing in the endoplasmic reticulm (ER) $[3,4]$. Wolframin is thought to be involved in the regulation of ER stress and calcium homeostasis, and Wolframin deficiency in mice leads to progressive loss of $\beta$ cells and impaired glucose tolerance, which is presumably caused by increased ER stress and apoptosis in the $\beta$ cells. Recently, another causative gene, CISD2, has been identified from the analysis of different families of patients with Wolfram syndrome, and these patients have been classified as belonging to the WFS2 group (MIM 604928) [2]. The

Correspondence: Daniel J Klionsky

Tel: 734-615-6556; Fax: 734-647-9702

E-mail: klionsky@umich.edu initial publication of the CISD2 gene reports that the Cisd 2 protein also localizes in the ER, suggesting that WFS2 is another ER-related disease [2].

On the other hand, because the clinical symptoms of Wolfram syndrome are often associated with mitochondrial disorders such as deafness, optic atrophy and psychiatric disorders, and because the affected tissues and organs in Wolfram syndrome have a high metabolic demand and most of the clinical manifestations of Wolfram syndrome are consistent with an ATP supply defect, an alternate hypothesis is that Wolfram syndrome is caused by mitochondrial disorder. Indeed, one Wolfram syndrome patient shows morphologically and biochemical abnormal mitochondria in a muscle biopsy [5]. However, no further biochemical evidence that supports this hypothesis had been reported. A recent paper in the journal Genes \& Development, however, has addressed this issue by generating and analyzing a knockout mouse in the WFS2 causative gene, Cisd2, demonstrating that one of the Wolfram syndromes, WFS2, is a mitochondrial-mediated disorder [6]. This finding helps resolve the apparent discrepancy between some of the clinical evidence and the localization of the causative gene product. The Cisd2 knockout (Cisd2 $2^{--}$) mouse demonstrates a premature aging phenotype, and detailed examination of this mouse provides interesting insight into the relation of neurodegenerative diseases, mitochondrial disorders and autophagy.

It is well accepted that mitochondrial dysfunction is related with premature aging. The mitochondrial DNA polymerase proofreading-deficient $\left(\right.$ Polg $A^{\text {mut } m u t}$ ) mouse is one of the wellcharacterized premature aging animal models where the phenotype is caused by mitochondrial dysfunction [7]. Mutated or deleted mitochondrial DNA accumulate in this mouse, which displays symptoms of premature aging such as weight loss, reduced subcutaneous fat, hair loss, lordokyphosis and osteopenia [7]. The Cisd $2^{-/-}$mouse shows early onset glucose intolerance and optic atrophy, typical symptoms of Wolfram syndrome, and also a premature aging phenotype similar to that observed in the mitochondrial-deficient ( $P \operatorname{olg} A^{\text {mut/ }}$ $\left.{ }^{m u t}\right)$ mouse. Histologically, the Cisd2 $2^{--}$ mouse shows skeletal and cardiac muscle and neuron degeneration that sometimes includes abnormal mitochondria, again similar to the Polg $A^{\text {mut } m u t}$ mouse. These findings suggest that the phenotype shown in the Cisd $2^{--}$mouse is caused by the mitochondrial deficiency. The Cisd 2 protein is localized on the mitochondrial outer membrane, and the mitochondria fractionated from skeletal muscle of the Cisd $2^{--}$mouse displays impaired mitochondrial respiratory 
chain function. These findings further support the idea that the phenotype of

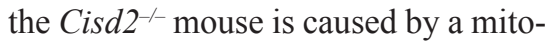
chondrial defect.

Interestingly, transmission electron microscopy analysis of the Cisd $2^{-/-}$ mouse shows a cluster of degenerating mitochondria and autophagic vacuoles in muscle cells and neurons. One biochemical marker of autophagy, the LC3II/LC3-I ratio, increases in muscles of the $C i s d 2^{-/-}$mouse, also suggesting the induction of autophagy. Because the damaged mitochondria induce autophagy as a cytoprotective response to eliminate the dysfunctional organelle, the sequestration of mitochondria into autophagosomes, which is observed in Cisd $2^{-/}$mouse muscle cells or neurons, is presumably the result of mitophagy (mitochondria-specific autophagy). The degenerating mitochondria observed in the Cisd2 $2^{--}$mouse sometimes maintain the structure of the inner cristae, although the outer membrane has lost integrity. These mitochondria may be destined for degradation by mitophagy, although it is not clear whether these organelles are present within autolysosomes. On the other hand, the presence of autophagic vacuoles in degenerating muscle cells or neurons provides morphological evidence of cell death with autophagic features.

Similar findings can be observed in typical neurodegenerative diseases such as Parkinson and Alzheimer disease. In the case of neurodegenerative diseases, it is unclear whether autophagy is induced for a cellular protective purpose that can degrade mutant or toxic proteins derived from neurodegeneration, or if autophagy is the cause of cell death that eventually leads to neurodegeneration. Recent studies have revealed a relation between neurodegenerative diseases and autophagy. For example, loss of autophagy in neural cells causes neurodegeneration in mice $[8,9]$. Also, Parkin, the Parkinson disease-related protein, is required for mitophagy to eliminate damaged mitochondria [10]. The findings from the Cisd $2^{--}$mouse and other neurodegenerative diseases suggest a strong correlation of mitochondrial dysfunction, autophagy (including cell death with autophagic features, and mitophagy) and neurodegeneration, although it is difficult to determine which is cause and effect. In the case of the Cisd2 $2^{--}$mouse, it may be reasonable to surmise that mitochondrial dysfunction induces autophagy (in particular mitophagy) to eliminate the damaged mitochondria, that the ATP depletion caused by mitochondrial elimination accelerates autophagy, and that the cells finally succumb to cell death with autophagic features.

With the characterization of the Cisd $2^{--}$mouse, it becomes clear that Wolfram syndrome is caused by at least two independent types of pathogenesis. The WFS1 group (MIM 606201) carrying a mutation of the WFS1 gene is caused by increased ER stress and subsequent apoptosis. On the other hand, the WFS2 group (MIM 604928) carrying a mutation of the CISD2 gene is caused by mitochondrial disorders. Because the $\mathrm{Cis} \mathrm{2}^{--}$mouse shows typical symptoms of Wolfram syndrome, and because this mouse shows a premature aging phenotype due to mitochondrial dysfunction but is not accompanied by the accumulation of mitochondrial DNA mutations (this is the first mouse model that shows mitochondrial-mediated premature aging without mitochondrial DNA mutations), this mouse is useful for the pathophysiological understanding of both Wolfram syndrome and mitochondrial-mediated premature aging.

\section{References}

1 Barrett TG, Bundey SE. Wolfram (DIDMOAD) syndrome. J Med Genet 1997; 34:838-841.

2 Amr S, Heisey C, Zhang M, et al. A homozygous mutation in a novel zincfinger protein, ERIS, is responsible for Wolfram syndrome 2. Am J Hum Genet 2007; 81:673-683.

3 Inoue H, Tanizawa Y, Wasson J, et al. A gene encoding a transmembrane protein is mutated in patients with diabetes mellitus and optic atrophy (Wolfram syndrome). Nat Genet 1998; 20:143148.

4 Strom TM, Hortnagel K, Hofmann $\mathrm{S}$, et al. Diabetes insipidus, diabetes mellitus, optic atrophy and deafness (DIDMOAD) caused by mutations in a novel gene (wolframin) coding for a predicted transmembrane protein. Hum Mol Genet 1998; 7:2021-2028.

5 Bundey S, Poulton K, Whitwell H, Curtis E, Brown IA, Fielder AR. Mitochondrial abnormalities in the DIDMOAD syndrome. J Inherit Metab Dis 1992; 15:315-319.

6 Chen YF, Kao CH, Chen YT, et al. Cisd2 deficiency drives premature aging and causes mitochondria-mediated defects in mice. Genes Dev 2009; 23:1183-1194.

7 Trifunovic A, Wredenberg A, Falkenberg M, et al. Premature ageing in mice expressing defective mitochondrial DNA polymerase. Nature 2004; 429:417-423.

8 Hara T, Nakamura K, Matsui M, et al. Suppression of basal autophagy in neural cells causes neurodegenerative disease in mice. Nature 2006; 441:885889.

9 Komatsu M, Waguri S, Chiba T, et al. Loss of autophagy in the central nervous system causes neurodegeneration in mice. Nature 2006; 441:880-884.

10 Narendra D, Tanaka A, Suen DF, Youle RJ. Parkin is recruited selectively to impaired mitochondria and promotes their autophagy. J Cell Biol 2008; 183:795-803. 